\title{
Consensus Problem of Heterogeneous Multi-agent Systems with Time Delay under Fixed and Switching Topologies
}

\author{
Ping-Ping Dai Cheng-Lin Liu Fei Liu \\ Key Laboratory of Advanced Process Control for Light Industry (Ministry of Education), Jiangnan University, Wuxi 214122, China
}

\begin{abstract}
Consensus problem is investigated for heterogeneous multi-agent systems composed of first-order agents and second-order agents in this paper. Leader-following consensus protocol is adopted to solve consensus problem of heterogeneous multi-agent systems with time-varying communication and input delays. By constructing Lyapunov-Krasovkii functional, sufficient consensus conditions in linear matrix inequality (LMI) form are obtained for the system under fixed interconnection topology. Moreover, consensus conditions are also obtained for the heterogeneous systems under switching topologies with time delays. Simulation examples are given to illustrate effectiveness of the results.
\end{abstract}

Keywords: Consensus problem, heterogeneous multi-agent systems, switching topologies, communication delay, input delay.

\section{Introduction}

In the past decade, consensus problem, which means that each agent reaches an agreement on a common value asymptotically by cooperating with their neighbors, has attracted much attention in several areas for its broad engineering applications, such as automated highway systems, air traffic control, congestion control in communication networks, etc.

In the multi-agent systems, each agent needs certain input delay for itself to accept and process information, and communication delays between neighboring agents are nonnegligible when the agents exchange information. So far, two types of consensus protocols with communication delays have been extensively adopted. One is that both the state of the agent and its neighbors are affected by identical delays, while another is that delays only affect the state received from neighbors of the agents.

Up to now, there has been much research on consensus problem of first-order ${ }^{[1-6]}$, second-order ${ }^{[7-19]}$, and highorder $^{[20,21]}$ multi-agent systems with identical agents' dynamics. In some practical situations, however, the agent of multi-agent systems has different dynamics for various constraints. Hence, consensus problem of heterogeneous multiagent systems has attracted many researchers' interests.

For heterogeneous multi-agent systems composed of firstorder and second-order integrator agents, Zheng et al. ${ }^{[22]}$ discussed the consensus problem under the linear consensus protocol and the saturated consensus protocol, and the sufficient conditions were established when the communication topologies were undirected connected graphs and leader-following networks. Zheng and Wang ${ }^{[23]}$ studied the finite-time consensus problem of heterogeneous multi-agent

Brief Paper

Manuscript received September 9, 2013; revised December 19, 2013

This work was supported by National Natural Science Foundation of China (Nos.61104092, 61134007 and 61203147), the Priority Academic Program Development of Jiangsu Higher Education Institutions. systems, and two classes of consensus protocols with and without velocity measurements were proposed by combining the homogeneous domination method with the addition of power integrator method. Liu and $\mathrm{Liu}^{[24]}$ investigated consensus problem for discrete-time heterogeneous multi-agent systems composed of first-order and secondorder agents, and obtained the sufficient consensus criteria for the agents with bounded communication delays under fixed topology and switching topologies, respectively. Moreover, Yin et al. ${ }^{[25]}$ investigated the consensus problem for a set of discrete-time heterogeneous multi-agent systems and designed a consensus algorithm based on the eventtriggered communication scheme, where communication is only needed between the agent and its local neighbors. In addition, a sufficient condition is obtained to guarantee the consensus of heterogeneous multi-agent systems in terms of linear matrix inequality (LMI) based on the Lyapunov functional method and the Kronecker product technique. Tian and Zhang ${ }^{[26]}$ studied the high-order consensus problem for heterogeneous multi-agent systems with unknown communication delays, and got a necessary and sufficient consensus condition. Munz et al. ${ }^{[27]}$ considered the multiagent systems with agents' dynamics given by strictly stable linear systems with nonuniform communication delays, and set-valued consensus conditions have been acquired based on the properties of the frequency-domain feedback matrix.

In this paper, a general stationary consensus protocol is adopted for heterogeneous multi-agent systems with input and communication delay to follow a static leader. Firstly, consensus is analyzed for the system with time-varying input delay and communication delay under fixed topology. By constructing Lyapunov-Krasovskii functional, sufficient consensus condition is obtained and expressed in LMI form. Secondly, a delay-dependent consensus condition in the form of LMI is also obtained for the system with timevarying input delay and communication delay converging to the leader's states under switching topologies. By using LMI toolbox in Matlab software, the bound of time delay 
can be determined based on LMI.

\section{Problem description}

In this paper, it is assumed that the heterogeneous multiagent system is composed of $m$ second-order agents and $n-m$ first-order agents. The dynamics of heterogeneous multiagent systems are given as

$$
\left\{\begin{array}{l}
\dot{x}_{i}(t)=v_{i}(t), \\
\dot{v}_{i}(t)=u_{i}(t), \quad i=1,2, \cdots, m \\
\dot{x}_{i}(t)=u_{i}(t), \quad i=m+1, \cdots, n
\end{array}\right.
$$

where $x_{i} \in \mathbf{R}, v_{i} \in \mathbf{R}$ are the position, velocity and $u_{i} \in \mathbf{R}$ is the control input respectively of agent $i$.

The interconnection topology of multi-agent systems is usually described as directed graph (digraph). Let $G=$ $(V, E, A)$ as the weighted digraph, $V=\{1, \cdots, n\}$ is a set of vertices, $E \subseteq V \times V$ is a set of edges, and $A=\left[a_{i j}\right] \in \mathbf{R}^{n \times n}$ is a weighted adjacency matrix such that $a_{i j}>0$ and $a_{i i}=0$ for all $i=1, \cdots, n$. The node indexes $n$ belong to a finite index set $\Lambda=\{1, \cdots, n\}$. The set of neighbors of node $i$ is denoted by $N_{i}=\{j \in V(i, j) \in E\}$. The Laplacian matrix of the weighted digraph $G$ is defined as $L=D-A$, where $D=\operatorname{diag}\left\{\sum_{j=1}^{n} a_{i j}, i \in \Lambda\right\}$ is the degree matrix of $G$.

Based on the protocols in $[8,15]$, we proposed a consensus protocol with a static leader as follows

$$
\begin{aligned}
u_{i}= & -k_{2} v_{i}(t)+k_{1} \sum_{j=1}^{n} a_{i j}\left(x_{j}(t)-x_{i}(t)\right)+ \\
& b_{i}\left(x_{0}-x_{i}(t)\right), \quad i=1,2, \cdots, m \\
u_{i}= & \sum_{j=1}^{n} a_{i j}\left(x_{j}(t)-x_{i}(t)\right)+b_{i}\left(x_{0}-x_{i}(t)\right), \\
& i=m+1, \cdots, n
\end{aligned}
$$

where $k_{1}>0, k_{2}>0, a_{i j}>0, j \in N_{i}$ is the adjacent element of neighbors matrix $A . x_{0} \in \mathbf{R}$ is a constant that denotes the position of the static leader, and the linked weight $b_{i}$ from agent $i$ to the leader is defined as

$$
\begin{cases}b_{i}>0, & \text { if agent is connected to the leader } \\ b_{i}=0, & \text { otherwise. }\end{cases}
$$

In this paper, the interconnection topology composed of the $n$ agents and the static leader is denoted by $\bar{G}$.

Under the time-varying input delay $\tau(t)>0$ and the communication delay $\eta(t)>0$ between each agent, the protocol (2) can be written as

$$
\begin{aligned}
u_{i}= & -k_{2} v_{i}(t-\tau(t))+k_{1} \sum_{j=1}^{n} a_{i j}\left(x_{j}(t-\eta(t)-\tau(t))-\right. \\
& \left.x_{i}(t-\tau(t))\right)+b_{i}\left(x_{0}-x_{i}(t)\right), \quad i=1,2, \cdots, m \\
u_{i}= & \sum_{j=1}^{n} a_{i j}\left(x_{j}(t-\eta(t)-\tau(t))-x_{i}(t-\tau(t))\right)+ \\
& b_{i}\left(x_{0}-x_{i}(t)\right), \quad i=m+1, \cdots, n .
\end{aligned}
$$

With the consensus algorithm (3), the closed-loop form of system (1) is

$$
\begin{aligned}
\dot{x}_{i}(t)= & v_{i}(t), \\
\dot{v}_{i}(t)= & -k_{2} v_{i}(t-\tau(t))+k_{1} \sum_{j=1}^{n} a_{i j}\left(x_{j}(t-\eta(t)-\tau(t))-\right. \\
& \left.x_{i}(t-\tau(t))\right)+b_{i}\left(x_{0}-x_{i}(t)\right), \quad i=1,2, \cdots, m, \\
\dot{x}_{i}(t)= & \sum_{j=1}^{n} a_{i j}\left(x_{j}(t-\eta(t)-\tau(t))-x_{i}(t-\tau(t))\right)+ \\
& b_{i}\left(x_{0}-x_{i}(t)\right), \quad i=m+1, \cdots, n .
\end{aligned}
$$

Define $\bar{x}_{i}(t)=x_{i}(t)-x_{0}$, and we can get

$$
\begin{aligned}
\dot{\bar{x}}_{s}(t)= & v_{s}(t), \\
\dot{v}_{s}(t)= & -k_{2} I_{m} v_{s}(t-\tau(t))+k_{1}\left[A_{s s} \bar{x}_{s}(t-\eta(t)-\tau(t))+\right. \\
& \left.A_{s f} \bar{x}_{f}(t-\eta(t)-\tau(t))-D_{s} \bar{x}_{s}(t-\tau(t))\right]-B_{s} \bar{x}_{s}(t), \\
\dot{\bar{x}}_{f}(t)= & A_{f s} \bar{x}_{s}(t-\eta(t)-\tau(t))+A_{f f} \bar{x}_{f}(t-\eta(t)-\tau(t))- \\
& D_{f} \bar{x}_{f}(t-\tau(t))-B_{f} \bar{x}_{f}(t)
\end{aligned}
$$

where $\bar{x}_{s}=\left[\bar{x}_{1}, \bar{x}_{2}, \cdots, \bar{x}_{m}\right], v_{s}=\left[v_{1}, v_{2}, \cdots, v_{m}\right]$, and $\bar{x}_{f}=\left[\bar{x}_{m+1}, \cdots, \bar{x}_{n}\right] . \quad A_{s s} \in \mathbf{R}^{m \times m}$ is the adjacency matrix of second-order agents between each other, $A_{f f} \in \mathbf{R}^{(n-m) \times(n-m)}$ is the adjacency matrix of firstorder agents between each other, $A_{s f} \in \mathbf{R}^{m \times(n-m)}$ and $A_{f s} \in \mathbf{R}^{(n-m) \times m}$ is adjacency matrix between first-order and second-order agents, and $B_{s}=\operatorname{diag}\left\{b_{1}, \cdots, b_{m}\right\}, B_{f}=$ $\operatorname{diag}\left\{b_{m+1}, \cdots, b_{n}\right\}$.

Then, system (5) can be rewritten as

$$
\dot{y}(t)=H_{1} y(t)+H_{2} y(t-\tau(t))+H_{3} y(t-\eta(t)-\tau(t))
$$

where

$$
\begin{gathered}
y^{\mathrm{T}}(t)=\left[\bar{x}_{s}(t), v_{s}(t), \bar{x}_{f}(t)\right]^{\mathrm{T}}, H_{1}=\left[\begin{array}{ccc}
0 & I_{m} & 0 \\
-B_{s} & 0 & 0 \\
0 & 0 & -B_{f}
\end{array}\right], \\
H_{2}=\left[\begin{array}{ccc}
0 & 0 & 0 \\
-k_{1} D_{s} & -k_{2} I_{m} & 0 \\
0 & 0 & -D_{f}
\end{array}\right]
\end{gathered}
$$

and

$$
H_{3}=\left[\begin{array}{ccc}
0 & 0 & 0 \\
-k_{1} A_{s s} & 0 & k_{1} A_{s f} \\
A_{f s} & 0 & A_{f f}
\end{array}\right] .
$$

Therefore, the consensus convergence problem of multiagent system (4) is equivalent to the asymptotic stability of system (6).

\section{Consensus analysis}

First of all, the assumptions about the input delay and communication delay are made as follows.

Assumption 1. $0 \leqslant \tau(t) \leqslant \sigma, 0 \leqslant \dot{\tau}(t) \leqslant \mu<1, \sigma>0$, $t \geqslant 0$.

Assumption 2. $0 \leqslant \eta(t) \leqslant h, 0 \leqslant \dot{\eta}(t) \leqslant d<1, h>0$.

In the proof of the main results, moreover, the following lemma is needed ${ }^{[28]}$.

Lemma 1. Assume that $0 \leqslant \eta(t) \leqslant h, h>0$ is piecewise continuous function. For arbitrary differentiable vector function $x(t):[-h, \infty) \rightarrow \mathbf{R}^{n}$ and arbitrary positive 
definite matrix $M \in \mathbf{R}^{n \times n}$, the following inequality is established:

$$
\begin{aligned}
& \left(\int_{t-\eta(t)}^{t} \dot{x}^{\mathrm{T}}(s) \mathrm{d} s\right) M\left(\int_{t-\eta(t)}^{t} \dot{x}(s) \mathrm{d} s\right) \leqslant \\
& h \int_{t-h}^{t} \dot{x}^{\mathrm{T}}(s) M \dot{x}(s) \mathrm{d} s, \quad t \geqslant 0 .
\end{aligned}
$$

\subsection{Fixed topology}

In this section, consensus is analyzed for system (4) with time-varying input delay and time-varying communication delay under fixed topology.

Theorem 1. $\tau(t)$ and $\eta(t)$ satisfy Assumptions 1 and 2, respectively, and the interconnection topology $\bar{G}$ of multiagent system (4) has the leader as a globally reachable node. Then, multi-agent system (4) achieves an asymptotic consensus such that $\lim _{t \rightarrow \infty} x_{i}(t)=x_{0}, \lim _{t \rightarrow \infty} v_{i}(t)=0$, $i \in \Lambda$, if the following LMI

$$
\left[\begin{array}{ccc}
M_{11} & M_{12} & M_{13} \\
& M_{22} & M_{23} \\
& * & M_{33}
\end{array}\right]<0
$$

holds, where

$$
\begin{aligned}
M_{11}= & H_{1}^{\mathrm{T}} P+P H_{1}+Q_{1}+\sigma H_{1}^{\mathrm{T}} R_{1} H_{1}+h H_{1}^{\mathrm{T}} R_{2} H_{1}- \\
& \sigma^{-1} R_{1} \\
M_{12}= & P H_{2}+\sigma H_{1}^{\mathrm{T}} R_{1} H_{2}+h H_{1}^{\mathrm{T}} R_{2} H_{2}+\sigma^{-1} R_{1} \\
M_{13}= & P H_{3}+\sigma H_{1}^{\mathrm{T}} R_{1} H_{3}+h H_{1}^{\mathrm{T}} R_{2} H_{3} \\
M_{22}= & (1-\mu)\left(Q_{2}-Q_{1}\right)+\sigma H_{2}^{\mathrm{T}} R_{1} H_{2}+h H_{2}^{\mathrm{T}} R_{2} H_{2}- \\
& \sigma^{-1} R_{1}-h^{-1} R_{2} \\
M_{23}= & \sigma H_{2}^{\mathrm{T}} R_{1} H_{3}+h H_{1}^{\mathrm{T}} R_{2} H_{3}+h^{-1} R_{2} \\
M_{33}= & (d+\mu-1) Q_{2}+\sigma H_{3}^{\mathrm{T}} R_{1} H_{3}+h H_{3}^{\mathrm{T}} R_{2} H_{3}- \\
& h^{-1} R_{2}
\end{aligned}
$$

and $P, Q_{j}, R_{j}, j=1,2,3$ are positive definite matrices with appropriate dimensions.

Proof. Choose a Lyapunov-Krasovskii functional for the system (6) as follows:

$$
\begin{aligned}
V(t)= & V_{1}(t)+V_{2}(t)+V_{3}(t) \\
V_{1}(t)= & y^{\mathrm{T}}(t) P y(t) \\
V_{2}(t)= & \int_{t-\tau(t)}^{t} y^{\mathrm{T}}(s) Q_{1} y(s) \mathrm{d} s+ \\
& \int_{t-\eta(t)-\tau(t)}^{t-\tau(t)} y^{\mathrm{T}}(s) Q_{2} y(s) \mathrm{d} s \\
V_{3}(t)= & \int_{-\sigma}^{0} \int_{t+\theta}^{t} \dot{y}^{\mathrm{T}}(s) R_{1} \dot{y}(s) \mathrm{d} s \mathrm{~d} \theta+ \\
& \int_{-h-\sigma}^{-\sigma} \int_{t+\theta}^{t} \dot{y}^{\mathrm{T}}(s) R_{2} \dot{y}(s) \mathrm{d} s \mathrm{~d} \theta .
\end{aligned}
$$

Calculating the derivative of $V(t)$, we get

$$
\begin{aligned}
\dot{V}(t)= & \dot{y}^{\mathrm{T}}(t) P y(t)+y^{\mathrm{T}}(t) P \dot{y}(t)+(1-\mu) y^{\mathrm{T}}(t-\tau(t)) \\
& \left(Q_{2}-Q_{1}\right) y(t-\tau(t))+(d+\mu-1) y^{\mathrm{T}}(t-\eta(t)- \\
& \tau(t)) Q_{2} y(t-\eta(t)-\tau(t))+\sigma \dot{y}^{\mathrm{T}}(t) R_{1} \dot{y}(t)- \\
& \sigma^{-1}\left(y^{\mathrm{T}}(t)-y^{\mathrm{T}}(t-\tau(t))\right) R_{1}(y(t)-y(t-\tau(t)))+ \\
& h \dot{y}^{\mathrm{T}}(t) R_{1} \dot{y}(t)-h^{-1}\left(y^{\mathrm{T}}(t-\tau(t))-y^{\mathrm{T}}(t-\eta(t)-\right. \\
& \tau(t))) R_{1}(y(t-\tau(t))-y(t-\eta(t)-\tau(t))) .
\end{aligned}
$$

Based on Lemma 1, we have

$$
\dot{V}(t) \leqslant \varphi^{\mathrm{T}}(t) M \varphi(t)
$$

where $\varphi^{\mathrm{T}}(t)=\left[y^{\mathrm{T}}(t), y^{\mathrm{T}}(t-\tau(t)), y^{\mathrm{T}}(t-\eta(t)-\tau(t))\right]$ and $M$ is defined as (7). Therefore, if (7) is established, the system (6) is asymptotically stable, i.e., the agents in system (4) achieve an asymptotic consensus.

In practice, the input delay between the controller and actuator usually changes relatively slowly, but the derivative of communication delay may be greater than one or unknown for various communication constraints. When $\tau(t)$ satisfies Assumption 1 and the derivative of $\eta(t)$ is not less than one or unknown, we choose the following LyapunovKrasovskii functional

$$
\begin{aligned}
V(t)= & y^{\mathrm{T}}(t) P y(t)+\int_{-\sigma}^{0} \int_{t+\theta}^{t} \dot{y}^{\mathrm{T}}(s) R_{1} \dot{y}(s) \mathrm{d} s \mathrm{~d} \theta+ \\
& \int_{-h-\sigma}^{0} \int_{t+\theta}^{t} \dot{y}^{\mathrm{T}}(s) R_{2} \dot{y}(s) \mathrm{d} s \mathrm{~d} \theta .
\end{aligned}
$$

Thus, we can obtain the following consensus condition.

Theorem 2. $\tau(t)$ satisfies Assumption 1, and $d \geqslant 1$, or the derivative of $\eta(t)$ is unknown, and the interconnection topology $\bar{G}$ of multi-agent system (4) has the leader as a globally reachable node. The agents in system (4) achieve an asymptotic consensus, i.e., $\lim _{t \rightarrow \infty} x_{i}(t)=x_{0}$, $\lim _{t \rightarrow \infty} v_{i}(t)=0, i \in \Lambda$, if the following LMI

$$
\left[\begin{array}{ccc}
M_{f 11} & M_{f 12} & M_{f 13} \\
& M_{f 22} & M_{f 23} \\
& * & M_{f 33}
\end{array}\right]<0
$$

holds, where

$$
\begin{aligned}
M_{f 11}= & H_{1}^{\mathrm{T}} P+P H_{1}+\sigma H_{1}^{\mathrm{T}} R_{1} H_{1}+(h+\sigma) H_{1}^{\mathrm{T}} R_{2} H_{1}+ \\
& \sigma^{-1} R_{1}-(h+\sigma)^{-1} R_{2} \\
M_{f 12}= & P H_{2}+\sigma H_{1}^{\mathrm{T}} R_{1} H_{2}+(h+\sigma) H_{1}^{\mathrm{T}} R_{2} H_{2}+\sigma^{-1} R_{1} \\
M_{f 13}= & P H_{3}+\sigma H_{1}^{\mathrm{T}} R_{1} H_{3}+(h+\sigma)+H_{1}^{\mathrm{T}} R_{2} H_{3}+ \\
& (h+\sigma)^{-1} R_{2} \\
M_{f 22}= & \sigma H_{2}^{\mathrm{T}} R_{1} H_{2}+(h+\sigma) H_{2}^{\mathrm{T}} R_{2} H_{2}-\sigma^{-1} R_{1} \\
M_{f 23}= & \sigma H_{2}^{\mathrm{T}} R_{1} H_{3}+(h+\sigma) H_{1}^{\mathrm{T}} R_{2} H_{3} \\
M_{f 33}= & \sigma H_{3}^{\mathrm{T}} R_{1} H_{3}+(h+\sigma) H_{3}^{\mathrm{T}} R_{2} H_{3}-(h+\sigma)^{-1} R_{2}
\end{aligned}
$$

and $P, Q_{j}, R_{j} j=1,2,3$ are positive definite matrices with appropriate dimensions.

The proof of Theorem 2 is similar to that of Theorem 1 , so we omit it to contain the length of this paper. 


\subsection{Switching interconnection topology}

The structure of the interconnection topology will change as time changes due to the addition of new links or the obstacles in links. For example, the communication range of agents is limited, or the agents change communication links and coupling weights. In this section, we investigate the consensus problem of heterogeneous multi-agent systems with switching topologies. Here, we assume that the number of switching topologies is finite. The set of topologies is defined as $\Omega=\left\{\bar{G}_{w}, w=1,2, \cdots, N\right\}$, and each digraph $\bar{G}_{w}$ has the leader as the globally reachable node. The system (4) with time-varying delays and switching topologies can be represented as

$$
\begin{aligned}
\dot{x}_{s}(t)= & v_{s}(t) \\
\dot{v}_{s}(t)= & -k_{2} I_{m}^{w} v_{s}(t-\tau(t))+k_{1}\left[A_{s s}^{w} x_{s}(t-\eta(t)-\tau(t))+\right. \\
& \left.A_{s f}^{w} x_{f}(t-\eta(t)-\tau(t))-D_{s}^{w} x_{s}(t-\tau(t))\right]+ \\
& B_{s}^{w}\left(x_{0} 1_{m}-x_{s}(t)\right) \\
\dot{x}_{f}(t)= & A_{f s}^{w} x_{s}(t-\eta(t)-\tau(t))+A_{f f}^{w} x_{f}(t-\eta(t)-\tau(t))- \\
& D_{f}^{w} x_{f}(t-\tau(t))-B_{f}^{w}\left(x_{f}(t)-x_{0} 1_{n}\right) .
\end{aligned}
$$

Taking the variable transformation $\bar{x}_{i}(t)=x_{i}(t)-x_{0}$, we can get

$$
\begin{aligned}
\dot{\bar{x}}_{s}(t)= & v_{s}(t) \\
\dot{v}_{s}(t)= & -k_{2} I_{m}^{w} v_{s}(t-\tau(t))+k_{1}\left[A_{s s}^{w} \bar{x}_{s}(t-\eta(t)-\tau(t))-\right. \\
& \left.A_{s f}^{w} \bar{x}_{f}(t-\eta(t)-\tau(t))-D_{s}^{w} \bar{x}_{s}(t-\tau(t))\right]-B_{s}^{w} \bar{x}_{s}(t) \\
\dot{\bar{x}}_{f}(t)= & A_{f s}^{w} \bar{x}_{s}(t-\eta(t)-\tau(t))+A_{f f}^{w} \bar{x}_{f}(t-\eta(t)-\tau(t))- \\
& D_{f}^{w} \bar{x}_{f}(t-\tau(t))-B_{f}^{w} \bar{x}_{f}(t) .
\end{aligned}
$$

System (9) can be rewritten as

$$
\dot{y}(t)=H_{1}^{w} y(t)+H_{2}^{w} y(t-\tau(t))+H_{3}^{w} y(t-\eta(t)-\tau(t))
$$

where

$$
\begin{gathered}
y^{\mathrm{T}}(t)=\left[\bar{x}_{s}(t), v_{s}(t), \bar{x}_{f}(t)\right]^{\mathrm{T}}, H_{1}^{w}=\left[\begin{array}{ccc}
0 & I_{m}^{w} & 0 \\
-B_{s}^{w} & 0 & 0 \\
0 & 0 & -B_{f}^{w}
\end{array}\right], \\
H_{2}^{w}=\left[\begin{array}{ccc}
0 & 0 & 0 \\
-k_{1} D_{s}^{w} & -k_{2} I_{m}^{w} & 0 \\
0 & 0 & -D_{f}^{w}
\end{array}\right],
\end{gathered}
$$

and

$$
H_{3}^{w}=\left[\begin{array}{ccc}
0 & 0 & 0 \\
-k_{1} A_{s s}^{w} & 0 & k_{1} A_{s f}^{w} \\
A_{f s}^{w} & 0 & A_{f f}^{w}
\end{array}\right] .
$$

Theorem 3. $\tau(t)$ and $\eta(t)$ satisfy Assumptions 1 and 2 , and the interconnection topology $G_{w}$ of multi-agent systems (8) has the leader as a globally reachable node. Then, multi-agent systems (8) reach an asymptotic consensus, i.e., $\lim _{t \rightarrow \infty} x_{i}(t)=x_{0}, \lim _{t \rightarrow \infty} v_{i}(t)=0, i \in \Lambda$, if the following LMIs

$$
\left[\begin{array}{ccc}
M_{s 11} & M_{s 12} & M_{s 13} \\
& M_{s 22} & M_{s 23} \\
& * & M_{s 33}
\end{array}\right]<0
$$

hold for $w=1,2, \cdots, N$, where

$$
\begin{aligned}
M_{s 11}= & H_{1}^{w \mathrm{~T}} P+P H_{1}^{w}+Q_{1}+Q_{2}+\sigma H_{1}^{\mathrm{T}} R_{1} H_{1}^{w}+ \\
& (h+\sigma) H_{1}^{w \mathrm{~T}} R_{2} H_{1}^{w}-\sigma^{-1} R_{1}-(h+\sigma)^{-1} R_{2} \\
M_{s 12}= & P H_{2}^{w}+\sigma H_{1}^{w \mathrm{~T}} R_{1} H_{2}+ \\
& (h+\sigma) H_{1}^{w \mathrm{~T}} R_{2} H_{2}^{w}+\sigma^{-1} R_{1} \\
M_{s 13}= & P H_{3}^{w}+\sigma H_{1}^{w \mathrm{~T}} R_{1} H_{3}^{w}+(h+\sigma)+H_{1}^{w \mathrm{~T}} R_{2} H_{3}^{w}+ \\
& (h+\sigma)^{-1} R_{2} \\
M_{s 22}= & (\mu-1) Q_{1}+\sigma H_{2}^{w \mathrm{~T}} R_{1} H_{2}^{w}+(h+\sigma) H_{2}^{w \mathrm{~T}} R_{2} H_{2}^{w}- \\
\sigma^{-1} R_{1} & \\
M_{s 23}= & \sigma H_{2}^{w \mathrm{~T}} R_{1} H_{3}^{w}+(h+\sigma) H_{1}^{w \mathrm{~T}} R_{2} H_{3}^{w} \\
M_{s 33}= & (d+\mu-1) Q_{2}+\sigma H_{3}^{\mathrm{T}} R_{1} H_{3}^{w}+ \\
& (h+\sigma) H_{3}^{w \mathrm{~T}} R_{2} H_{3}^{w}-(h+\sigma)^{-1} R_{2}
\end{aligned}
$$

and $P, Q_{j}, R_{j}, j=1,2,3$ are positive definite matrices with appropriate dimensions.

Proof. Choosing a Lyapunov-Krasovskii functional for system (10)

$$
\begin{aligned}
V(t)= & V_{1}(t)+V_{2}(t)+V_{3}(t), \\
V_{1}(t)= & y^{\mathrm{T}}(t) P y(t), \\
V_{2}(t)= & \int_{t-\tau(t)}^{t} y^{\mathrm{T}}(s) Q_{1} y(s) \mathrm{d} s+ \\
& \int_{t-e t a(t)-\tau(t)}^{t} y^{\mathrm{T}}(s) Q_{2} y(s) \mathrm{d} s, \\
V_{3}(t)= & \int_{-\sigma}^{0} \int_{t+\theta}^{t} \dot{y}^{\mathrm{T}}(s) R_{1} \dot{y}(s) \mathrm{d} s \mathrm{~d} \theta+ \\
& \int_{-h-\sigma}^{0} \int_{t+\theta}^{t} \dot{y}^{\mathrm{T}}(s) R_{2} \dot{y}(s) \mathrm{d} s \mathrm{~d} \theta .
\end{aligned}
$$

Calculating the derivative of $V(t)$, we get

$$
\begin{aligned}
\dot{V}(t)= & \dot{y}^{\mathrm{T}}(t) P y(t)+y^{\mathrm{T}} P \dot{y}(t)+y^{\mathrm{T}}(t)\left(Q_{1}+Q_{2}\right) y(t)+ \\
& (\mu-1) y^{\mathrm{T}}(t-\tau(t)) Q_{1} y(t-\tau(t))+(d+\mu-1) y^{\mathrm{T}} \\
& (t-\eta(t)-\tau(t)) Q_{2} y(t-\eta(t)-\tau(t))+\sigma \dot{y}^{\mathrm{T}}(t) \\
& R_{1} \dot{y}(t)-\sigma^{-1}\left(y^{\mathrm{T}}(t)-y^{\mathrm{T}}(t-\tau(t))\right) R_{1}(y(t)- \\
& y(t-\tau(t)))+(h+\sigma) \dot{y}^{\mathrm{T}}(t) R_{2} \dot{y}(t)- \\
& (h+\sigma)^{-1}\left(y^{\mathrm{T}}(t-\tau(t))-y^{\mathrm{T}}(t-\eta(t)-\tau(t))\right) \\
& R_{2}(y(t-\tau(t))-y(t-\eta(t)-\tau(t))) .
\end{aligned}
$$

From Lemma 1, we have

$$
\dot{V}(t) \leqslant \varphi^{\mathrm{T}}(t) M \varphi(t)
$$

where $\varphi^{\mathrm{T}}(t)=\left[y^{\mathrm{T}}(t), y^{\mathrm{T}}(t-\tau(t)), y^{\mathrm{T}}(t-\eta(t)-\tau(t))\right]$ and $M$ is defined as (11). Therefore, if (11) is established, the system (10) is asymptotically stable, i.e., the agents in system (8) reach an asymptotic consensus.

Remark 1. If $\tau(t)$ satisfies Assumption 1 , and $d \geqslant 1$, or the derivative of $\eta(t)$ is unknown, we can use the same Lyapunov-Krasovskii functional as that in Theorem 2. to get the consensus condition for the system with switching topologies, but we omit it here to avoid repetition. 


\section{Simulation results}

In this paper, we assume that the multi-agent system is composed of two second-order agents and three first-order agents and a static leader, and the leader is the globally reachable node of the topology.

Example 1. The interconnection topology of system (4) is given in Fig. 1 . The node 0 is the static leader, and the nodes 1,2 and the nodes $3,4,5$ denote second-order agents and first-order agents respectively. We assume that the weights of edges are: $a_{12}=1, a_{23}=0.2, a_{34}=0.2, a_{45}=$ $0.3, a_{52}=0.3, b_{5}=1.5$. and the control gains are chosen as $k_{1}=0.1$ and $k_{2}=1$. The position of static leader is chosen as $x_{0}=1$, and the initial position and velocity of each node are generated randomly.

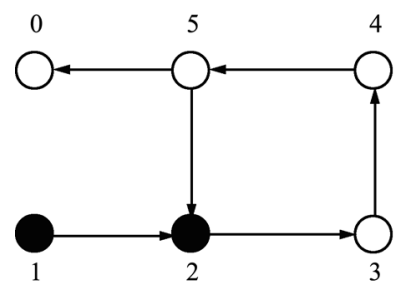

Fig. 1 Interconnection topology with five agents and a leader

1) The input delay and communication delay are timevarying. We choose $\eta(t)=6|\sin (0.1 t)|(\mathrm{s})$, and the derivative of $\eta(t)$ is less than 1 that satisfies Assumption 1. By using the LMI toolbox in Matlab for the LMI (7) in Theorem 1, we can get $\tau<0.478 \mathrm{~s}$. In this section, we assume that $\tau(t)=0.3|\sin (t)|(\mathrm{s})$, and the simulation results are shown in Fig. 2. Thus, each agent converges to the leader's state, i.e., $\lim _{t \rightarrow \infty} x_{i}(t)=x_{0}, i=1,2, \cdots, 5$.
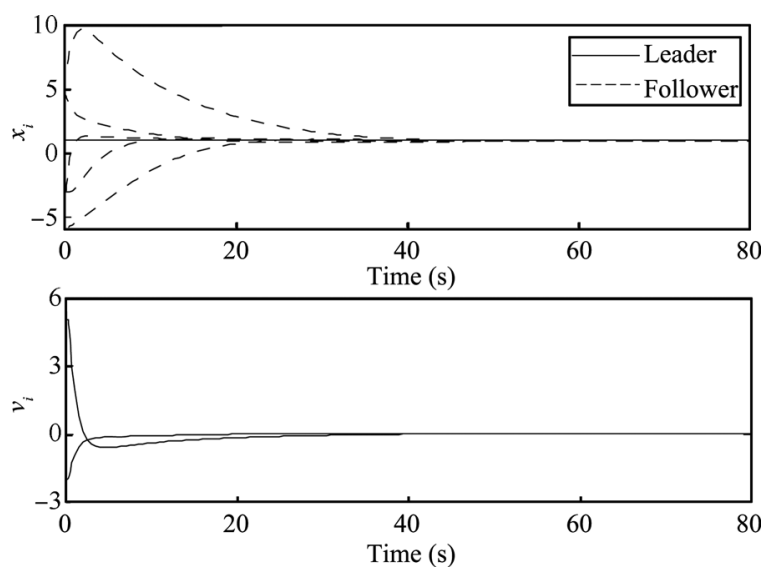

Fig. 2 Agents with $d<1$ under fixed topology

2) We choose $d>1$ and $\eta(t)=6|\sin (t)|$ (s) that satisfies Theorem 2. From the condition (7), we can get $\tau<0.417 \mathrm{~s}$. Choosing $\tau(t)=0.3|\sin (t)|(\mathrm{s})$, and we obtain that each agent converges to the leader's state, i.e., $\lim _{t \rightarrow \infty} x_{i}(t)=$ $1, i=1,2, \cdots, 5$ and $\lim _{t \rightarrow \infty} v_{i}(t)=0, i=1,2$. The simulation results are shown in Fig. 3.
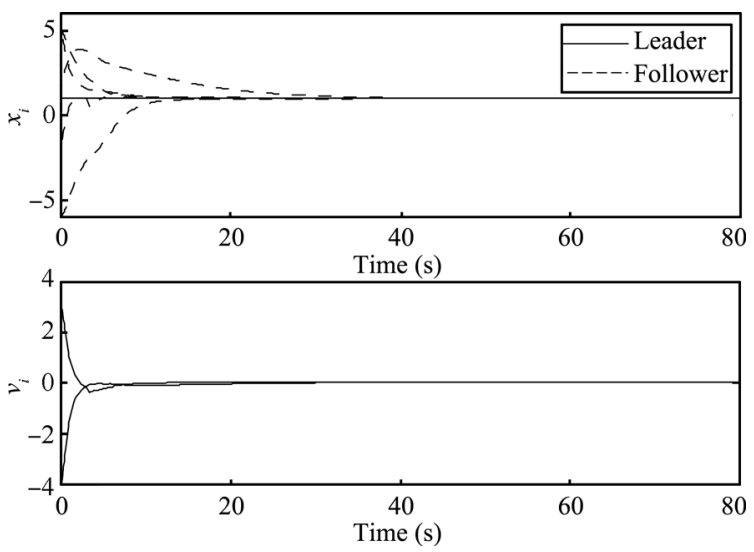

Fig. 3 Agents with $d>1$ under fixed topology

Example 2. Consider a network of five agents and a static leader given by (8). The interconnection topology of the system is switched between the topology 1 in Fig. 4 and the topology 2 in Fig. 5. In the simulation, the interconnection topology is switched from topology 1 to topology 2 every $5 \mathrm{~s}$. In topology 1 , we choose the weights as: $a_{12}=$ $0.2, a_{32}=0.3, a_{42}=0.05, a_{45}=0.05, a_{52}=0.3, b_{2}=1.2$. In topology 2 , we choose the weights as: $a_{15}=0.1, a_{21}=$ $0.1, a_{32}=0.3, a_{43}=0.1, a_{53}=0.3, b_{1}=0.8$. Topology 1 and topology 2 has the same control gains that are given by $k_{1}=0.2$ and $k_{2}=1$.

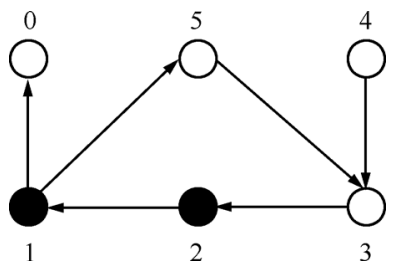

Fig. 4 Topology 1

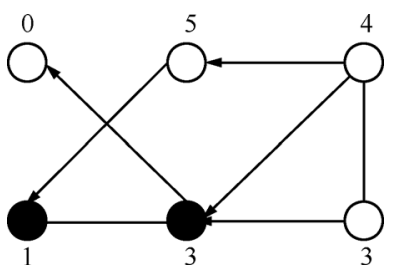

Fig. 5 Topology 2

1) Consider the input delays and communication delays are time-varying. Choose $\eta(t)=6|\sin (0.02 \pi t)|(\mathrm{s})$ and the derivative $d<1$ satisfying Assumption 1. By using the LMI toolbox in Matlab for the LMI (11) in Theorem 2, we get $\tau<0.812 \mathrm{~s}$. In this section, we assume $\tau(t)=0.3|\sin (0.5 \pi t)|(\mathrm{s})$, and we obtain that each agent in system (8) converges to the leader's state, i.e., $\lim _{t \rightarrow \infty} x_{i}(t)=x_{0}, i=1,2, \cdots, 5$ and $\lim _{t \rightarrow \infty} v_{i}(t)=0$, $i=1,2$. The simulation results are shown in Fig. 6 .

2) Choose $\eta(t)=6|\sin (2 t)|(\mathrm{s})$ that satisfies $d>1$. From condition (11), we can get $\tau<0.79 \mathrm{~s}$. By choosing $\tau(t)=0.3|\sin (0.5 \pi t)|(\mathrm{s})$, the closed-loop system (8) achieves an asymptotic consensus, i.e., $\lim _{t \rightarrow \infty} x_{i}(t)=1$, $\lim _{t \rightarrow \infty} v_{i}(t)=0, i=1,2, \cdots, 5$. The simulation results are shown in Fig. 7. 

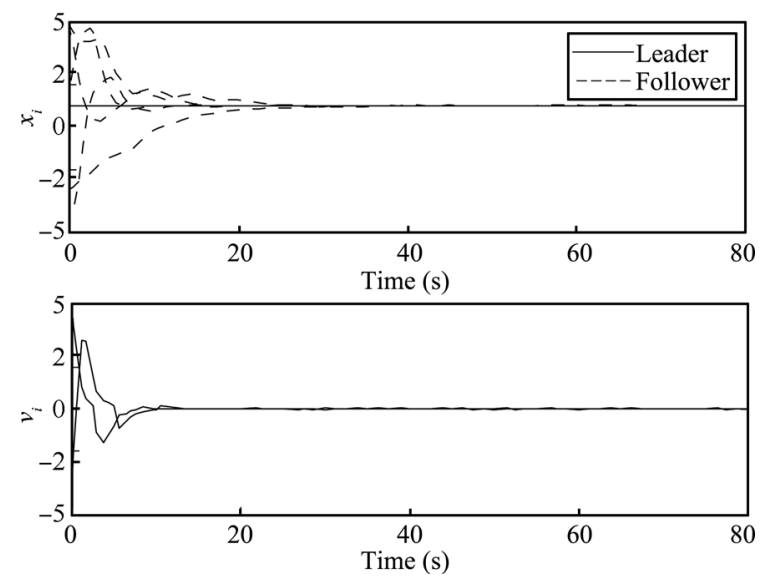

Fig. 6 Agents with $d<1$ under switching topologies
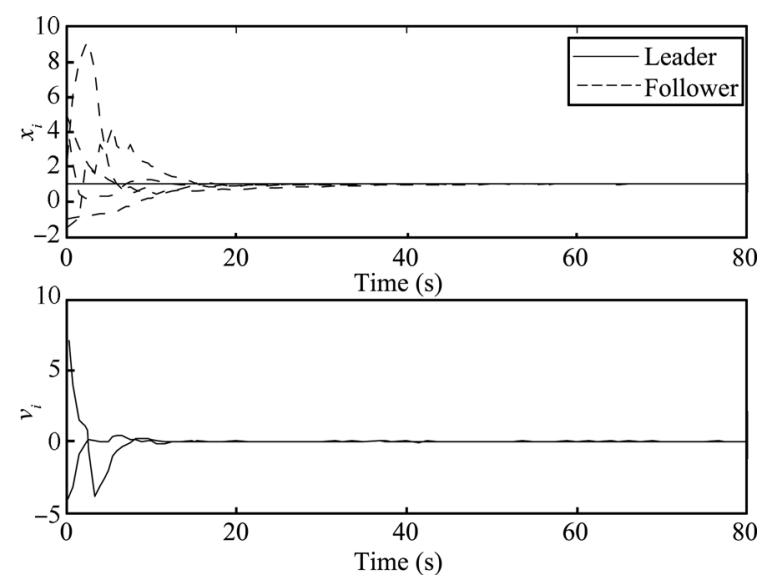

Fig. 7 Agents with $d>1$ under switching topologies

\section{Conclusion}

In this paper, a stationary leader-following consensus protocol is adopted to solve the consensus problem for heterogeneous multi-agent systems composed of first-order and second-order agents with time-varying input delay and communication delay under fixed topology and switching topologies. By constructing Lyapunov-Krasovskii functional, the sufficient consensus conditions are provided for the heterogeneous multi-agent systems with time-varying input delay and communication delay under fixed and switching topologies respectively. Furthermore, consensus conditions are obtained for unknown or different values of the derivative of communication delay. Although the consensus conditions are all formulated in the form of LMIs, the main results provide consensus criteria to obtain the time delay upper-bounds by using the LMI tool box in Matlab.

\section{References}

[1] R. Olfati-Saber, R. M. Murray. Consensus problems in networks of agents with switching topology and time-delays. IEEE Transactions on Automatic Control, vol.49, no.9, pp. 1520-1533, 2004.

[2] H. B. Du, S. H. Li, S. H. Ding. Bounded consensus algorithms for muliti-agent systems in directed networks. Asian Journal of Control, vol. 15, no. 1, pp. 282-291, 2013.
[3] F. Xiao, L. Wang. Asynchronous consensus in continuoustime multiagent systems with switching topology and timevarying delays. IEEE Transactions on Automatic Control, vol. 53, no. 8, pp. 1804-1816, 2006.

[4] F. C. Jiang, L. Wang. Finite-time information consensus for multi-agent systems with fixed and switching topologies. Physica D, vol. 238, no. 16, pp. 1550-1560, 2009.

[5] Y. K. Zhu, X. P. Guan, X. Y. Luo. Finit-time consensus for multi-agent systems via nonlinear control protocols. International Journal of Automation and Computing, vol. 10, no. 5, pp. 455-462, 2013.

[6] P. Lin, K. Y. Qin, H. M. Zhao, M. Sun. A new approach to average consensus problems with multiple time-delays and jointly-connected topologies. Journal of the Franklin Institute, vol. 349, no. 1, pp. 293-304, 2012.

[7] J. P. Hu, Y. G. Hong. Leader-following coordination of multi-agent systems with coupling time delays. Physica A, vol. 374, no. 2, pp. 853-863, 2007.

[8] J. H. Qin, W. X. Zheng, H. J. Gao. Consensus of multiple second-order vehicles with a time-varying reference signal under directed topology. Automatica, vol. 479, no. 9, pp. 1983-1991, 2011.

[9] J. Y. Yu, L. Wang. Group consensus in multi-agent systems with switching topologies and communication delays. Systems \& Control Letters, vol. 59, no. 6, pp. 340-348, 2010.

[10] H. Y. Yang, F. C. Wang, S. Y. Zhang. Consensus of secondorder multi-agent systems with nonsymmetric interconnection and heterogeneous delays. International Journal of $\mathrm{Au}$ tomation and Computing, vol. 8, no. 4, pp. 421-428, 2011.

[11] P. Lin, Y. M. Jia. Consensus of a class of secondorder multi-agent systems with time-delay and jointlyconnected topologies. IEEE Transactions on Automatic Control, vol. 55, no. 3, pp. 778-784, 2010.

[12] Y. P. Gao, L. Wang. Asynchronous consensus of continuoustime multi-agent systems with intermittent measurement. International Journal of Robust and Nonlinear Control, vol. 83, no. 3, pp. 552-562, 2010.

[13] C. L. Liu, F. Liu. Dynamical consensus seeking of secondorder multi-agent systems based on delayed state compensation. Systems \& Control Letters, vol. 61, no. 12, pp. 1235$1241,2012$.

[14] C. L. Liu, F. Liu. Consensus problem of second-order multiagent systems with time-varying communication delay and switching topology. Journal of Systems Engineering and Electronics, vol. 22, no. 4, pp.672-678, 2011.

[15] P. Lin, Y. M. Jia. Consensus of second-order discrete-time multi-agent systems with nonuniform time-delays and dynamically changing topologies. Automatica, vol.45, no. 9, pp. 2154-2158, 2006.

[16] S. H. Lin, H. B. Du, X. Z. Lin. Finite-time consensus algorithm for multi-agent systems with double-integrator dynamics. Automatica, vol. 47, no. 8, pp. 1706-1712, 2011.

[17] Y. P. Gao, J. W. Ma, M. Zuo, T. Q. Jiang, J. P. Du. Consensus of discrete-time second-order agents with time-varying topology and time-varying delays. Journal of the Franklin Institute, vol. 349, no. 8, pp. 2598-2608, 2012.

[18] W. G. Zhang, J. Z. Liu, D. L. Zeng, Y. Hu. Consensus of second-order multi-agent systems with nonuniform time delays. Chinese Physics B, vol. 22, no.5, Article 050511, 2013.

[19] M. J. Park, O. M. Kwon, J. H. Park, S. M. Lee, E. J. Cha. Leader-following consensus criteria for multi-agent systems with time-varying delays and switching interconnection topologie. Chinese Phys B, vol.21, no. 11, Article 110508, 2012. 
[20] T. Yang, Y. H. Jin, W. Wang, Y. J. Shi. Consensus of highorder continuous-time multi-agent systems with time-delays and switching topologies. Chinese Physics B, vol. 32, no. 1, Article 020511, 2011.

[21] Z. Q. Wu, Y. Wang. Dynamic consensus of high-order multiagent systems and its application in the motion control of multiple mobile robots. International Journal of Automation and Computing, vol. 9, no. 1, pp. 54-62, 2012.

[22] Y. Zheng, Y. Zhu, L. Wang. Consensus of heterogeneous multi-agent systems. IET Control Theory and Applications, vol. 15, no. 16, pp. 1881-1888, 2011.

[23] Y. S. Zheng, L. Wang. Finite-time consensus of heterogeneous multi-agent systems with and without velocity measurements. Systems \& Control Letters, vol.61, no. 8, pp. 871-878, 2012 .

[24] C. L. Liu, F. Liu. Stationary consensus of heterogeneous multi-agent systems with bounded communication delays. Automatica, vol. 47, no. 9, pp. 2130-2133, 2011.

[25] X. X. Yin, D. Yue, S. L. Hu. Distributed event-triggered control of discrete-time heterogeneous multi-agent systems. Journal of the Franklin Institute, vol. 350, no. 3, pp.651669, 2013.

[26] Y. P. Tian, Y. Zhang. High-order consensus of heterogeneous multi-agent systems with unknown communication delays. Automatica, vol. 48, no. 6, pp. 1205-1212, 2012.

[27] U. Munz, A. Papachristodoulou, F. Allgower. Delay robustness in consensus problem. Automatica, vol. 46, no. 8, pp. 1252-1265, 2010.

[28] K. Q. Gu. An integral inequality in the stability problem of time-delay systems. In Proceedings of the 39th IEEE Conference on Decision and Control, IEEE, Sydney, Australia, pp. 2805-2810, 2000.

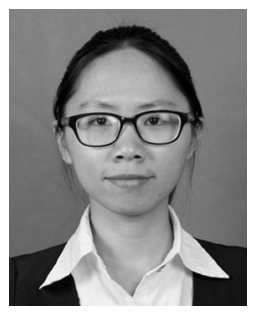

Ping-Ping Dai received the B. Sc. degree in automation from Jiangnan University, China in 2011. Since September 2011 she has been studying in Jiangnan University for the M. Sc. degree.

Her research interest includes the coordination control of multi-agent systems.

E-mail: joepingdai@gmail.com

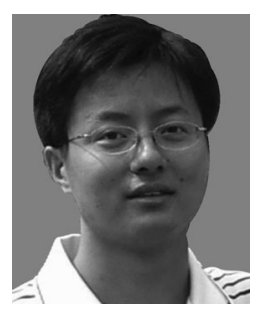

Cheng-Lin Liu received the B.Sc. degree in electrical engineering and automation from Nanjing University of Science and Technology, China in 2003. He received his $\mathrm{Ph}$. D. degree in control theory and control engineering at Southeast University, China in 2008. He is currently a faculty member at Institute of Automation, Jiangnan University, China.

His research interests include Internet congestion control and coordination control of multi-agent systems.

E-mail: liucl@jiangnan.edu.cn (Corresponding author)

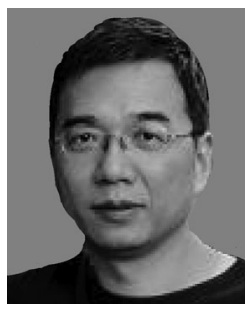

Fei Liu received the $\mathrm{Ph} . \mathrm{D}$. degree in control science and control engineering from Zhejiang University, China. Now, he is a professor of the Institute of Automation, Jiangnan University, China.

His research interests include advanced control theory and applications, batch process control engineering, statistical monitoring and diagnosis in industrial process, and intelligent technique with emphasis on fuzzy and neural systems.

E-mail: fliu@jiangnan.edu.cn 